\title{
Electrical Conductivity of a Semidilute Polyelectrolyte in Mixed Solvent Media
}

\author{
Ajaya Bhattarai \\ Department of Chemistry, Mahendra Morang Adrash Multiple Campus,Biratnagar \\ Tribhuvan University, Nepal \\ email:bkajaya@yahoo.com
}

\begin{abstract}
The electrical conductivity of solutions of sodium salt of polystyrenesulfonate in methanol-water mixed solvent media containing $8,16,25$, and 34 weight per cent of methanol at $308.15,313.15,318.15$ and $323.15 \mathrm{~K}$ was studied earlier. In that report, an attempt was made to interprete the results using the Manning Counterion condensation theory. Unfortunately, a major deviation of the theoretical prediction from the experimental results was noticed and the possible reasons for this discrepancy have been discussed. In this communication, the experimental conductivity have been analyzed on the basis of a new model for semi-dilute polyelectrolyte conductivity which takes into electrical conductivity of semi-dilute solutions of polyelectrolytes without added salt has been recently described by Colby et al.(1997) using the scaling description proposed by Dobrynin et al.(1995) for the configuration of a polyelectrolyte chain in semi-dilute solution. The predictions are found to be compared favorably well with the observed conductivity over the whole range of concentrations investigated.
\end{abstract}

Key Words: conductivity, counterion, condensation, semi-dilute, Manning theory

\section{Introduction}

Polyelectrolytes are polymers with ionizable groups. When they are dissolved in aqueous or mixed solvent these groups dissociate, leaving ions on the chain and counterions in the solution. The specific conductance and the equivalent conductivity, $\Lambda$, are experimentally determined parameters which are suitable to describe the electrolytic transport properties of polyelectrolyte solutions because these properties take into account the movement of any charged entity present in the system under the influence of an externally applied electric field.

Inspite of various attempts by different investigators, a completely satisfactory theory to describe the electrolytic conductivity of polyelectrolyte solutions has not yet been developed (Mandel 1988, Leeuven et al. 1991, Wandrey 1997).

However, the description of different electrical properties of polyions in aqueous solutions and of their interactions with counterions is generally based on the Manning counterion condensation theory (Manning a.b $1969,1975,1981)$ that, under some aspects, can be considered equivalent to the Poisson-Boltzmann cylindrical cell model (Katchalsky 1971, Stigter 1975, Mandel 1992, Le Bret \& Zimm. 1984). Within this model, the polyion is represented as an infinitely long charged line, small ions (counterions) are assumed to form an ionic atmosphere whose density depends on the frame of the polyion and their interactions with the charged polyions groups are purely Coulombic, so that the screening effect extends over the Debye length. The uncondensed mobile ions are treated in the DebyeHückel approximation. The solvent is assumed to be a continuous medium characterized by a spatially uniform dielectric constant. Interactions among polyions are neglected, the theory being addressed to highly diluted solutions. The basic idea is that if the charge density $(\xi)$ of the rod exceeds a critical value, some charges on the chain can be partially neutralized by one of the free ions in the solution, which means that the repulsion Coulombic energy of two adjacent unit charges on the chain must be smaller than the thermal energy $k_{B} T$. On the basis of these assumptions, this approach has been successfully applied to different polyelectrolyte systems, and numerous experiments have strongly suggested the validity of this model (Shaaban et al. 1994, Penafiel \& Litovitz 1992, Kuznetsov et al. 1991, Kwak \& Hayes 1975, Szymczac et al. 1975, Kozak et al. 1971, Kwak et al. 1978). 
Nepal Journal of Science and Technology 9(2008)163-170

According to the Manning counterion condensation theory, polyelectrolytes can be characterized by the linear charge density parameter defined by (Manning 1969, 1972, Oosawa 1971)

$$
\xi=\frac{e^{2}}{b D k_{B} T}
$$

where $e$ is the protonic charge, $D$ the relative permitivity of the medium, $b$ the spacing between charged groups taken along the axis of the polyion chain, $k_{\mathrm{B}}$ the Boltzman constant and $T$ the temperature in absolute scale. This theory states that if $\xi>1$, enough counterions condense on to the polyion to yield the critical value $\xi=1$. If, on the other hand, $\xi<1$, ionization takes place to reach this critical value. A limiting law of electrical conductivity for a salt free polyelectrolyte solution is related to $\xi$ by the following equation (Manning 1969, 1972, 1970)

$$
\Lambda=f\left(\lambda_{c}^{0}+\lambda_{\mathrm{p}}\right)
$$

where $\lambda_{\mathrm{c}}^{0}$ and $\lambda_{\mathrm{p}}$ are the limiting ionic equivalent conductivity of the counterion and the equivalent conductivity of the polyion at a finite concentration, respectively, and $f$ is counterion-polyion interaction parameter given by(Manning 1969,1972,Oosawa 1971)

$f=\frac{0.866}{\xi}$

In contrast to Huizenga et al. 1950 where the counterions are regarded either as "bound" or as "free" implying that they are not influenced by the polyion, Manning assumed that while the condensed counterions, certainly qualify as bound, the fraction of such ions is $1-\xi^{-1} \neq 1-f$, the uncondensed counterions may, in no way, be thought of as free, being subjected to the Debye-Hückel potential of the polyions. Thus $f$ does not have the significance of free counterions rather it represents the fraction of uncondensed counterions. Considering electrophoretic and relaxation contributions to the equivalent conductivity, $\lambda_{\mathrm{p}}$ has been derived theoretically for the cylinder model of polyelectrolyte (Manning 1975), and it follows for counterions with a charge of $z_{\mathrm{c}}$

$$
\lambda_{\mathrm{p}}=\frac{279 A\left|z_{\mathrm{c}}\right|^{-1}|\ln \kappa a|}{1+43.2 A\left(\left|z_{\mathrm{c}}\right| \lambda_{\mathrm{c}}^{0}\right)^{-1}|\ln \kappa a|}
$$

where the parameter $a$ is the radius of the polymer chain, while

$A=\frac{D k_{B} T}{3 \pi \eta_{0} e}$

with $\eta_{0}$ being the coefficient of viscosity of the solvent. In eq. $4, \kappa$ is the Debye screening constant, which is defined by

$\kappa^{2}=\frac{4 \pi \cdot e^{2}}{D k_{\mathrm{B}} T} \xi^{-1} n_{\mathrm{e}}\left|z_{\mathrm{c}}\right|$

where $n_{\mathrm{e}}$ is the stoichiometric equivalent polyion concentration (monovalent charged groups per unit volume).

\section{Materials and Methods}

Methanol (E. Merck, India, 99\% pure) was distilled with phosphorous pentoxide and then redistilled over calcium hydride. The purified solvent had a density of $0.7772 \mathrm{~g} . \mathrm{cm}^{-3}$ and a co-efficient of viscosity of $0.4742 \mathrm{mPa}$.s at $308.15 \mathrm{~K}$; these values are in good agreement with the literature values (Moumouzias et al.1991). Triply distilled water with a specific conductance less than $10^{-6}{\mathrm{~S} . \mathrm{cm}^{-1}}^{\text {at }} 308.15 \mathrm{~K}$ was used for the preparation of the mixed solvents. The physical properties of methanol-water mixed solvents used in this study at $308.15,313.15,318.15$, and $323.15 \mathrm{~K}$ are reported in Table 1. The relative permittivity of methanol-water mixtures at the experimental temperatures were obtained by regressing the relative permittivity data as function of solvent composition from the literature (Albright \& Gasting 1946) and are included in Table 1.

Sodium polystyrenesulphonate (NaPSS) employed in these investigations was purchased from Aldrich Chemical Company, Inc. The average molecular weight $\left(M_{\mathrm{w}}\right)$ of the sample was $c a .70,000$. 
A. Bhattarai./Electrical Conductivity of A Semidilute...............

Table 1. Properties of Methanol-Water Mixtures Containing 8, 16, 25, and 34 Weight Per Cent of Methanol at 308.15, $313.15,318.15$, and $323.15 \mathrm{~K}$ and the Corresponding $\lambda_{r}^{0}$ Values of the Sodium Ion

\begin{tabular}{|c|c|c|c|c|}
\hline $\mathrm{T} / K$ & $\rho_{0} / \mathrm{g} \cdot \mathrm{cm}^{-3}$ & $\eta_{0} / \mathrm{mPa} . \mathrm{s}$ & D & $\lambda_{\mathrm{c}}^{0} / \mathrm{S} \mathrm{cm}^{2} \cdot \mathrm{mol}^{-1}$ \\
\hline \multicolumn{5}{|c|}{8 per cent methanol } \\
\hline 308.15 & 0.9797 & 0.8665 & 71.57 & 58.12 \\
\hline 313.15 & 0.9780 & 0.7752 & 69.99 & 63.86 \\
\hline 318.15 & 0.9760 & 0.7017 & 68.18 & 71.06 \\
\hline 323.15 & 0.9741 & 0.6375 & 66.45 & 79.52 \\
\hline \multicolumn{5}{|c|}{16 per cent methanol } \\
\hline 308.15 & 0.9663 & 1.0217 & 68.14 & 53.32 \\
\hline 313.15 & 0.9641 & 0.9091 & 66.63 & 60.88 \\
\hline 318.15 & 0.9616 & 0.8075 & 64.80 & 69.65 \\
\hline 323.15 & 0.9588 & 0.7300 & 63.15 & 76.78 \\
\hline \multicolumn{5}{|c|}{25 per cent methanol } \\
\hline 308.15 & 0.9516 & 1.1418 & 64.25 & 49.76 \\
\hline 313.15 & 0.9490 & 1.0084 & 62.81 & 57.01 \\
\hline 318.15 & 0.9463 & 0.8957 & 60.99 & 64.06 \\
\hline 323.15 & 0.9433 & 0.8052 & 59.41 & 73.55 \\
\hline \multicolumn{5}{|c|}{34 per cent methanol } \\
\hline 308.15 & 0.9310 & 1.2034 & 60.34 & 47.52 \\
\hline 313.15 & 0.9300 & 1.0503 & 58.94 & 52.85 \\
\hline 318.15 & 0.9254 & 0.9309 & 57.18 & 59.20 \\
\hline 323.15 & 0.9234 & 0.8288 & 55.62 & 66.02 \\
\hline
\end{tabular}

Table 2. Best Fitted ' $A$ ' in Methanol-Water Mixtures at 308.15, 313.15, 318.15 K, and 323.15K; respective standard deviation value $(\mathrm{sd})$

\begin{tabular}{|c|c|c|c|c|c|c|c|c|}
\hline \multirow{2}{*}{$\begin{array}{l}\mathrm{wt} \% \text { of } \\
\text { methanol }\end{array}$} & \multicolumn{2}{|c|}{$T=308.15 \mathrm{~K}$} & \multicolumn{2}{|c|}{$T=313.15 \mathrm{~K}$} & \multicolumn{2}{|c|}{$T=318.15 \mathrm{~K}$} & \multicolumn{2}{|c|}{$T=323.15 \mathrm{~K}$} \\
\hline & hol sd & best fitted"A" & $\mathrm{sd}$ & t fitted"A & ' sd & t fitted"A' & sd bes & fitted"A" \\
\hline 8 & 2.4446 & 3.86 & 3.6811 & 4.30 & 2.4888 & 4.18 & 3.7284 & 4.00 \\
\hline 16 & 2.9481 & 3.95 & 3.7697 & 4.25 & 3.1757 & 4.19 & 3.7563 & 4.21 \\
\hline 25 & 2.3839 & 4.15 & 2.4309 & 4.35 & 3.2640 & 4.40 & 3.0669 & 4.42 \\
\hline 34 & 1.1401 & 4.22 & 2.0968 & 4.43 & 2.2782 & 4.53 & 1.9132 & 4.71 \\
\hline
\end{tabular}


Nepal Journal of Science and Technology 9(2008)163-170

Conductance measurements were carried out on a Pye-Unicam PW 9509 conductivity meter at a frequency of $2000 \mathrm{~Hz}$ using a dip-type cell with a cellconstant of $1.15 \mathrm{~cm}^{-1}$ and having an uncertainty of $0.01 \%$. The cell was calibrated by the method of Lind and co-workers (Lind et al. 1957) using aqueous potassium chloride solution. The measurements were made in a water bath maintained within $\pm 0.005 \mathrm{~K}$ of the desired temperature. The details of the experimental procedure have been described earlier (Das \& Hazra 1992, 1995).Several independent solutions were prepared and runs were performed to ensure the reproducibility of the results. Due correction was made for the specific conductance of the solvent by subtracting the specific conductance of the relevant solvent medium from those of the polyelectrolyte solutions.

In order to avoid moisture pickup, all solutions were prepared in a dehumidified room with utmost care. In all cases, the experiments were performed at least in three replicates.

\section{Results and Discussion}

It is noteworthy that the Manning theory does not contain any adjustable parameter, and despite its simplicity, the main results based on this model (Manning a, b 1969, Mandel et al.1984, de Gennes 1980) are fairly well satisfied by the experimental data mainly for colligative properties, although they are valid only at infinite dilution. Since the Manning theory applies, as stated above, to highly diluted systems where polyion-polyion interactions are assumed to be absent and to polyions modeled as a linear array of point charges, its validity is limited to very low concentration regimes of polyelectrolyte solutions. Up to now, the electrical conductivity has been repeatedly measured for a great variety of polyelectrolytes (Bhattarai et al. 2006, Nagasawa et al. 1972, Kwak et al. 1975, Kwak and Johnston, 1975, Szymczak et al. 1975, Kwak et al. 1978, Vink 1984, Rios et al. 1993, Abramovic and Klofutar 1997, Rios 2001, Liu et al.1999, Wandrey 1999). In most of these cases, the concentrations are far from being very dilute and primarily the semidilute solutions (c>c*) have been studied. The application of the Manning model to these systems is, however, less straightforward because these semi-dilute macroions do not assume a fully stretched conformation in solution. Even then some studies (Kwak \& Hayes1975) reported close agreement between experiment and the Manning theory in the semi-dilute regime. However, these surprising results should not be over interpreted, since the underlying reason is nothing but a fortuitous cancellation of two opposing contributions of approximately the same size, which are not contained in the limiting law. Careful measurements (Bhattarai et al. 2006, Kwak and Hayes 1975, Szymczak et al. 1975, Rios et al. 1993, Abramovic and Klofutar 1997, Rios 2001, Liu et al. 1999) of the electrical conductivity of aqueous salt-free polyelectrolyte solutions demonstrated a major deviation of the experimental results from the Manning theory.

A new model for the electrical conductivity of semidilute solutions of polyelectrolytes without added salt has been recently proposed by (Colby et al. 1997) using the scaling description proposed by (Dobrynin et al. 1995) for the configuration of a polyelectrolyte chain in semi-dilute solution.

In the semi-dilute solutions, the polyion chain is modeled as a random walk of $N_{\xi}$ correlation blobs of size $\xi_{0}$, each of them containing $g$ monomers. Each blob bears an electric charge $q_{\xi}=z e f g$ ( $z$ being the counterion valence and $e$ is the electronic charge) and the complete chain, of contour length $L=N_{\xi} \xi_{0}$, bears a charge $Q_{\mathrm{p}}=N_{\xi} q_{\xi}=z e f g N_{\xi}$. Due to the strong electrostatic interactions within each correlation blob, the chain is a fully extended conformation of $g_{\mathrm{e}}$ electrostatic blobs of size $\xi_{\mathrm{e}}$. This means that for length scales less than $\xi_{0}$, the electrostatic interactions dominate (and the chain is a fully extended conformation of electrostatic blobs of size $\xi_{\mathrm{e}}$ ), and for length scales greater than $\xi_{0}$, the hydrodynamic interactions are screened and the chain is a random walk of correlation blobs of size $\xi_{0}$.

Within this model, in absence of added salt, the equivalent conductivity of a polyion in a semi-dilute solution is given by

$$
\lambda_{\mathrm{p}}=\frac{F z e f g \ln \left(g / g_{e}\right)}{3 \pi \eta_{0} \xi_{0}}
$$

where $F$ is the Faraday number and $\eta_{0}$ is the coefficient of viscosity of solvent.

Under the condition of $g / g_{\mathrm{e}}=\xi_{0} / \xi_{\mathrm{e}}$ and making use of the fact that the electrostatic blobs are space filling ( $g=c \xi_{0}^{3}, c$ being the number density of monomers) Eq. 7 reduces to

$$
\lambda_{\mathrm{p}}=\frac{F z e f c \xi_{0}^{2} \ln \left(\xi_{0} / \xi_{e}\right)}{3 \pi \eta_{0}}
$$




\section{A. Bhattarai./Electrical Conductivity of A Semidilute.}

The equivalent conductance of the polyelectrolyte solution is then given by

$\Lambda=f\left[\lambda_{c}{ }^{0}+\frac{F z e f c \xi_{0}^{2} \ln \left(\xi_{0} / \xi_{e}\right)}{3 \pi \eta_{0}}\right]$

The above expression given by (Colby et al. 1997 ) is derived taking into account the scaling arguments proposed by ( Dobrynin et al. 1995) neglecting the hydrodynamic interactions (Kirkwood model) between the single structural units i.e., the correlation blobs and neglecting the asymmetry field effect. As can be seen the above model has a single free parameter $f$, the fraction of uncondensed counterions that defines the degree of the effectively ionized groups along the chain, in the scheme of the Manning counterion condensation theory. Whereas the Manning theory applies to polyelectrolyte solutions in the highly diluted regime and predicts a fraction of condensed counterions independent of the polymer concentration given by the

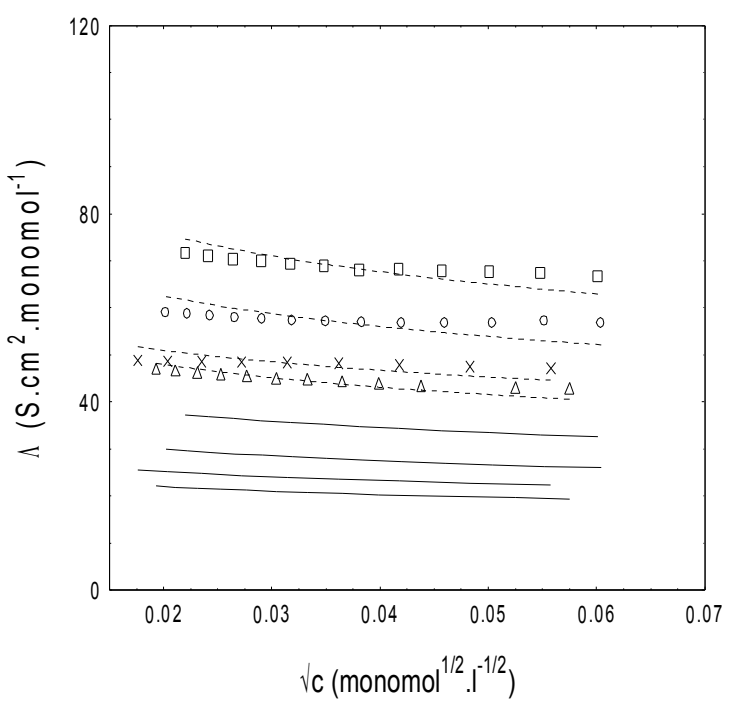

Fig.1. Comparison of the experimental values of the equivalent conductivity $(\Lambda)$ of solutions of sodium polystyrenesulphonate with those predicted on the basis of the Manning counterion condensation theory and on the basis of the scaling theory at $308.15 \mathrm{~K}$ : Experimental values: squares, 8 per cent methanol; circles, 16 per cent methanol; crosses, 25 per cent methanol, triangles, 34 per cent methanol; Manning theory : solid lines for 8, 16, 25 and 34 per cent methanol respectively from top to bottom; Scaling theory with best fitted A: broken lines. value of the charge-density parameter $\xi=\lambda_{\mathrm{B}} / b$. This model, which is based on the scaling theory approach, has been applied, so far, to a limited number of aqueous polyelectrolytes(Bordi et al. 2004 ) and the good agreement with the experimental conductivity results is very encouraging.

Here we present a study on the electrical conductivity of the semi-dilute solutions of sodium polystyrenesulfonate in methanol-water mixed solvent media at four different temperatures keeping in mind the failure of the Manning model in explaining our experimental findings (Bhattarai et al. 2006) we analyzed our experimental data according to the model derived from the scaling concept. This is, in fact, the first report on the application of the scaling theory approach to semi-dilute polyelectrolyte conductivity in mixed solvent media. The influence of concentration, medium, and temperature on the mobility of the polyelectrolyte species will also be investigated.

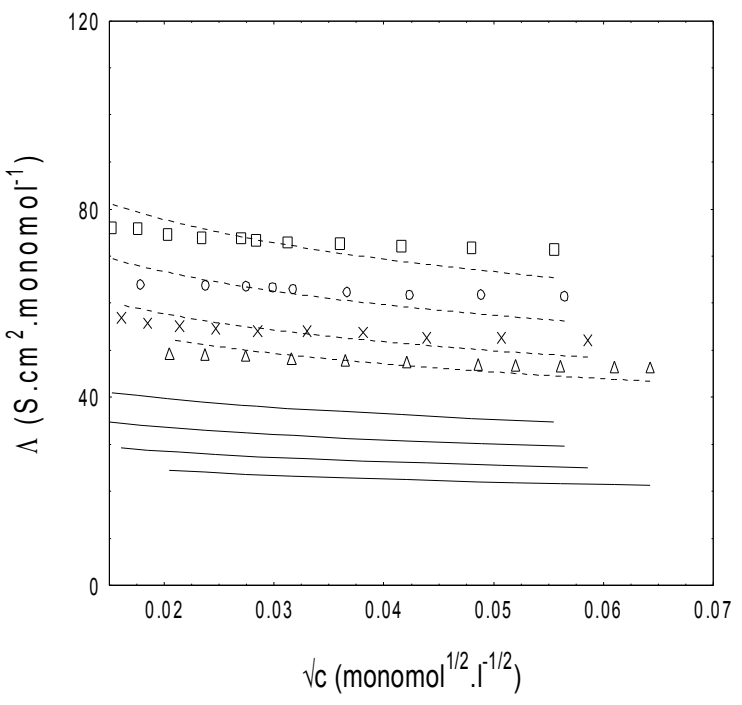

Fig. 2. Comparison of the experimental values of the equivalent conductivity $(\Lambda)$ of solutions of sodium polystyrenesulphonate with those predicted on the basis of the Manning counterion condensation theory and on the basis of the scaling theory at $313.15 \mathrm{~K}$ : Experimental values: squares, 8 per cent methanol; circles, 16 per cent methanol; crosses, 25 per cent methanol, triangles, 34 per cent methanol; Manning theory : solid lines for 8 , 16,25 and 34 per cent methanol respectively from top to bottom; Scaling theory with best fitted A: broken lines. 
Nepal Journal of Science and Technology 9(2008)163-170

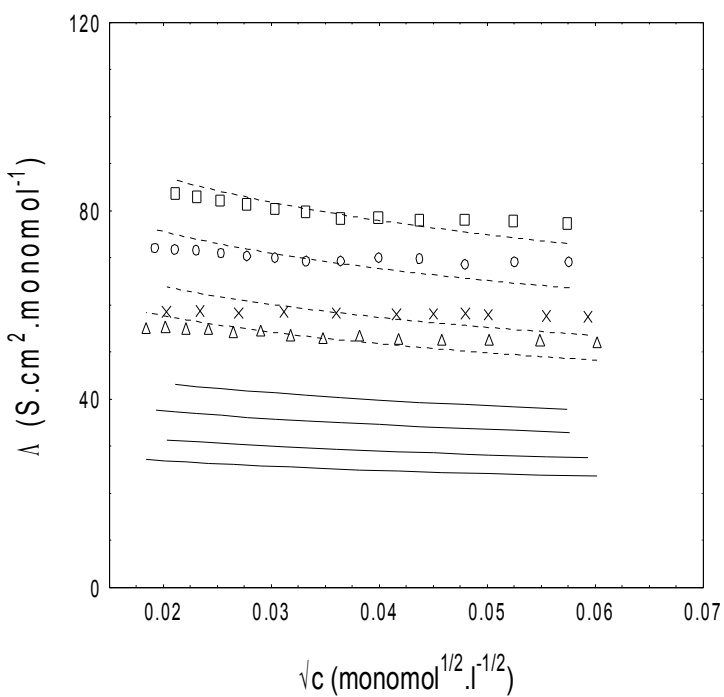

Fig. 3. Comparison of the experimental values of the equivalent conductivity $(\Lambda)$ of solutions of sodium polystyrenesulphonate with those predicted on the basis of the Manning counterion condensation theory and on the basis of the scaling theory at $318.15 \mathrm{~K}$ : Experimental values: squares, 8 per cent methanol; circles, 16 per cent methanol; crosses, 25 per cent methanol, triangles, 34 per cent methanol; Manning theory : solid lines for 8, 16, 25 and 34 per cent methanol respectively from top to bottom; Scaling theory with best fitted A: broken lines.

Graphical representation of the experimental $\Lambda$ values (points), the Manning predictions (solid lines) and calculated values based on Colby model values (broken lines) are included in Figures $1-4$ at 308.15, 313.15, 318.15 and $323.15 \mathrm{~K}$ in methanol-water mixtures. The experimentally obtained equivalent conductivities have always been found to be considerably higher than the theoretical values calculated following the Manning model. The reason for the failure of the Manning model can be understood if one estimates the overlap concentration $\left(c^{*}\right)$ for the polymer chain investigated using the following equation (Wandrey and Langmuir 1999)

$c^{*}=\frac{1}{N_{\mathrm{A}} L^{2} a}$

where $L$ is the contour length and the other symbols have their usual significance. It is observed that the polymer solutions in the present study are essentially in the semi-dilute regime where the Manning limiting law does not apply.

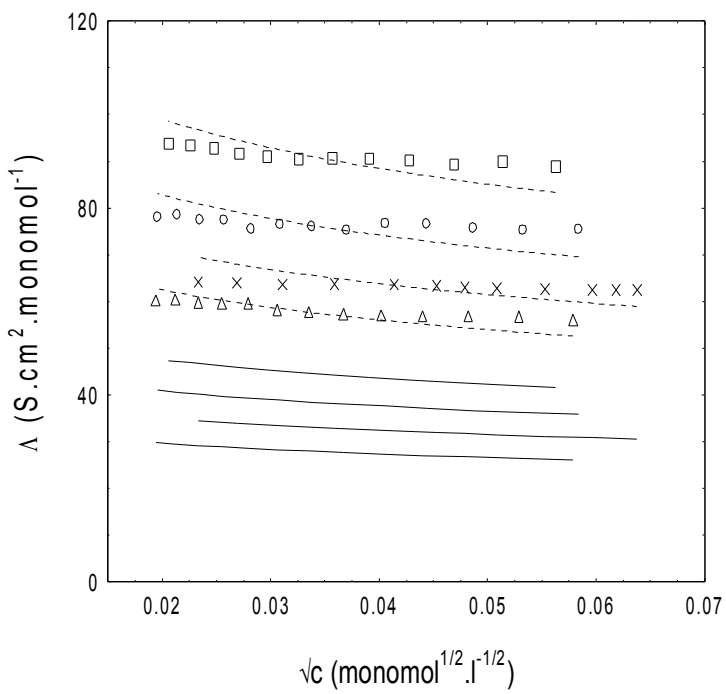

Fig. 4. Comparison of the experimental values of the equivalent conductivity $(\Lambda)$ of solutions of sodium polystyrenesulphonate with those predicted on the basis of the Manning counterion condensation theory and on the basis of the scaling theory at $323.15 \mathrm{~K}$ : Experimental values: squares, 8 per cent methanol; circles, 16 per cent methanol; crosses, 25 per cent methanol, triangles, 34 per cent methanol; Manning theory : solid lines for 8, 16,25 and 34 per cent methanol respectively from top to bottom; Scaling theory with best fitted A: broken lines.

We, therefore, have taken the recourse of the approach proposed by (Colby et al. 1997) for semi-dilute polyelectrolyte solutions, for analysis of our conductivity data.

Since the present methanol-water mixtures are poor solvent for the uncharged polymer sodium polystyrenesulfonate, the electrostatic blob is collapsed into a dense globule, we use a value of $8 \mathrm{~A}^{0}$ as the effective monomer size $(b)$ as suggested by (Colby et al. 1997) while analyzing the data on the basis of the Colby model (Colby et al. 1997).

Under poor solvent condition, the electrostatic blob size $\left(\xi_{\mathrm{e}}\right)$ and the correlation blob size $\left(\xi_{0}\right)$ are given by (Colby et al. 1997)

$\xi_{e}=b\left(\frac{A^{2} b}{\lambda_{B}}\right)^{1 / 3}$

and 
A. Bhattarai./Electrical Conductivity of A Semidilute.

$\xi_{0}=(c b)^{-1 / 2}\left(\frac{A^{2} b}{\lambda_{B}}\right)^{1 / 3}$

It is interesting to note that the equivalent conductivity calculated here is found to be highly sensitive on the change in ' $A$ ' value in equation 11 and a very good agreement with the experimental result is obtained for an optimum value of ' $A$ '. The best fit ' $A$ ' values which describe the experimental results best along with the respective standard deviation of fits are given in Table 2. We have, thus, been able to quantitatively describe the electrical conductivity data of the Sodium polystyrenesulfonate in methanol-water mixed solvent media with the help of the scaling concept of polyelectrolyte solutions.

An investigation on electrical conductivity of salt-free solution of an anionic polyelectrolyte sodium polystyrenesulfonate in methanol-water mixed solvent media has been presented as a function of polymer concentration and temperature. The equivalent conductivities are found to increase with increasing temperature over the entire concentration range in a given mixed solvent medium whereas these values are found to decrease as the relative permittivity of the medium decreases. The conductivity theory, proposed by Manning, for salt-free polyelectrolyte solutions was applied previously to analyze the experimental data. The measured values of equivalent conductivity could not be quantitatively described by this theory. A recent model proposed by Colby et al. based on scaling approach for the configuration of a polyelectrolyte chain in semidilute solution has also been tested and the results derived from this model are found to be quantitatively consistent with the experimental observations. The influences of temperature and the relative permittivity of the medium on the equivalent conductivity were also investigated.

\section{References}

Abramovic, H. and C.Klofutar. 1997. Conductivity of some tetra-n-alkylammonium salts of pectic acid. Eur. Polym. J. 33:1295-1300.

Albright,P.S. and L. J.Gasting.1946.Dielectric constant of the methanol-water system from 5 to $55^{\circ} \mathrm{c} . \mathrm{J} . \mathrm{Am}$. Chem. Soc. 68:1061-1063.

Bhattarai, A., P.Nandi and B. Das. 2006. The Effects of concentration, relative permittivity and temperature on the transport properties of sodium polystyrenesulphonate in methanol-water mixed solvent media. J. Pol. Res. 13:475-482.

Bordi,F.,C. Cammetti and R. H. Colby. 2004.Dielectric spectroscopy and conductivity of polyelectrolyte solutions. J. Phys. Condens. Matter. 16:1423-1463.

Colby,R.H.,D. C. Boris,W. E. Krause and J.S.Tan.1997 Polyelectrolyte conductivity. J. Polym. Sci. Part B. 35:2951-2960.

Das,B.andD.K. Hazra. 1992. Studies on the viscosities, conductance and adiabatic compressibilities of some tetraalkylammonium perchlorates in 2-methoxyethanol. Bull. Chem. Soc. Jpn. 65:3470-3476.

Das,B.andD.K.Hazra.1995.Conductometric,viscometric, and spectroscopic investigations on the solvation phenomena of alkali-metal ions and ion pairs in 2methoxyethanol. J. Phys. Chem. 99: 269-273.

Dobrynin, A. V., R. H.Colby and M.Rubinstein.1995. Scaling theory of polyelectrolyte solutions. Macromolecules, 28:1859-1871.

de Gennes,P.G.1980.Scaling Concepts in Polymer Physics. Cornell University Press: Ithaca, New York.

Katchalsky,A.1971.Polyelectrolytes. Pure Appl. Chem. 2: 327-373.

Kozak, D., J. Kristan and D. Dolar.1971. Osmotic coefficient of polyelectrolyte solutions.I. Polystyrenesulphonates with monovalent counterions. $Z$. Phys. Chem. (NF) 76:85-92.

Kuznetsov, I. A., O. A. Vorontsova and A. G. Kozlov.1991. Polyelectrolyte properties of biopolymers: Conductivity and secondary structure of polyriboadenylic acid and its salts in solutions. Biopolymers.31:65-76.

Kwak, J. C. T., F. Murphy and E.Spiro.1978. The equivalent conductivity of aqueous solutions of alkali metal salts of a number of ionic polysaccharides. J. Biophys. Chem. 7:379-386.

Kwak, J. C. T. and R. C. Hayes.1975. Electrical conductivity of aqueous solutions of salts of polystyrenesulfonic acid with univalent and divalent counterions. J. Phys. Chem. 79: 265-269.

Kwak, J. C. T. and A .J. Johnston.1975.The Equivalent conductivity of aqueous solutions of salts of carboxymethylcellulose: A Test of Manning's limting law. Can. J. Chem. 53:792-796.

Le Bret, M.and B. H.Zimm.1984.Distribution of counterions around a cylindrical polyelectrolyte and manning's condensation theory. Biopolymers.23:287-312.

Leeuven, H. P.V., R. F. M. Cleven and P.Valenta. 1991. Conductometric analysis of polyelectrolytes in Solution. Pure Appl. Chem. 63:1251-1268.

Liu, S., H. Jia, D. Yang and Ji. Faxiang. 1999. Electrical conductivity and counterion association of cationic polyacrylamide derivatives in aqueous solution. Polym. Int. 48:1080-1084.

Lind, Jr. J. E., J. J.Zwolenik and R. M Fuoss. 1959. Calibration of conductance cells at $25^{\circ}$ with aqueous 
Nepal Journal of Science and Technology 9(2008)163-170

solutions of potassium chloride.J. Am. Chem. Soc. 81:1557-1559.

Mandel, M. 1992. The Poisson-Boltzmann equation for aqueous solutions of strong polyelectrolytes without added salt: the cell model revisited.$J$. Phys. Chem. 96:3934-3942.

Mandel, M. 1988. In Encyclopedia of Polymer Science and Engineering, (2nd ed.)( Eds Mark, H. F., N. M. Bikales, C. G Overberger, G.Menges) Wiley \& Sons: New York 739pp.

Mandel, M and T.Odijk.1984. Dielectric properties of polyelectrolyte solutions. Annu. Rev. Phys. Chem. 35:75-108.

Manning,G. S. 1969a. Limiting laws and counterion condensation in polyelectrolyte solutions I. colligative properties. .J. Chem. Phys. 51: 924-933.

Manning,G. S. 1969b.Limiting laws and counterion condensation in polyelectrolyte solutions II. Selfdiffusion of the Small ions. J. Chem. Phys. 51: 934 938.

Manning,G.S.1975.A Limiting law for the conductance of the rod model of a salt-free polyelectrolyte solution. J. Chem. Phys. 79: 262-265.

Manning,G. S. 1981.Limiting laws and counterion condensation in polyelectrolyte solutions. 7. electrophoretic mobility and conductance.J. Chem. Phys. 85: 1506-1515.

Manning, G. S. 1972. Polyelectrolytes. Ann. Rev. Phys. Chem. 23: 117-140.

Manning,G.S.1970.On the interpretation of conductance measurements in salt-free polyelectrolyte solutions with an application to the helix-coil transition of poly (D-glutamic acid).Biopolymers.9:1543-1546. Moumouzias,G.D.,K.PanopoulosandG.Ritzoulis.1991. Excess properties of the binary liquid system propylene carbonate+acetonitrile.J.Chem Eng. Data. 36: 20-23.

Nagasawa,M.,I.Noda,T.Takahashi\&N.Shimamoto. 1972 .Transport phenomena of polyelectrolytes in solution under electric field.J. Phys. Chem. 76:2286-2294.

Oosawa,F.1971.Polyelectrolyte,MarcelDekker,NewYork

Penafiel, L. M. and T. A. Litovitz .1992. Counterion condensation and ionic conductivity.J. Chem. Phys. 96:3033-3038.

Rios, H. E. 2001.Electrical conductivity properties of poly [(maleic acid)-co-(vinyl pyrrolidone)] salts in aqueous solutions. Polym. Int. 50:885-890.

Rios, H. E., R. G. Barraza and I. C. Gamboa.1993. Polyelectrolyte solutions. Electrical conductivity and counterion condensation. Polym. Int. 31:213-217.

Stigter, D.1975. The Charged colloidal cylinder with a Gouy double layer. J Colloid Interface Sci., 53:296-306.

Shaaban, A. H., K.Behilo and P.Ander. 1994. In Macro-ion Characterization: From Dilute Solutions to Complex Fluids; K. S. Schmitz, Ed.; ACS Symposium Series 548; American Chemical Society: Washington, DC.

Szymczak, J., P.Holyk and P.Ander.1975. Electrical conductivity of aqueous solutions of monovalent salts of polystyrenesulfonate. J. Phys. Chem. 79:269-272.

Vink, H. 1984. Electrolytic conductivity of multicomponent polyelectrolyte solutions. J. Chem. Soc., Faraday Trans.l.80: 507-514.

Wandrey,C.1999.Concentration Regimes in polyelectrolyte Solutions. Langmuir 15:4069-4075.

Wandrey,C.1997.Polyelektrolyte-Makromolekulare Parameter und Elektrolyt Verhalten;CuvillierVerlag:Go“ttingen, Germany. 